\title{
The ATLAS/TileCal Detector Control System
}

\author{
H. Santos, on behalf of the ATLAS Tile Calorimeter Group
}

\begin{abstract}
The main task of the Tile calorimeter Detector Control System (DCS) is to enable the coherent and safe operation of the calorimeter. All actions initiated by the operator, as well as all errors, warnings and alarms concerning the hardware of the detector are handled by DCS. The Tile calorimeter DCS controls and monitors mainly the low voltage and high voltage power supply systems, but it is also interfaced with the infrastructure (cooling system and racks), the calibration systems, the data acquisition system, configuration and conditions databases and the detector safety system.
\end{abstract}

Index Terms-LHC, ATLAS, TILECAL, Detector Control System, PVSS II, Finite State Machine

\section{INTRODUCTION}

$\mathbf{T}$ HE Tile Calorimeter (TileCal) is one of the sub-detectors of the ATLAS experiment. It is a sampling calorimeter made of steel plates (absorber) and scintillating tiles (active material) readout by wavelength shifting optical fibers. The photodetectors are photomultiplier tubes (PMTs). The design, general features and expected performance of the calorimeter can be found in [1]. The TileCal consists of one barrel and two extended barrel parts. These three sections are further sub-divided into 4 partitions - EBA, LBA, LBC and EBC (standing EB and LB for Extended Barrel and Long Barrel, respectively). Each partition is a cylinder sub-divided into 64 independent modules. The calorimeter has 5184 cells, $97.2 \%$ of which are operational (in May, 2010). The cells are defined by grouping together sets of optical fibers into bunches leading to the PMTs and each cell has redundant reading by two PMTs. Part of the Front End (FE) electronics and PMTs are located at the rear of the TileCal modules, in the so-called electronics drawers.

The detector itself is located $100 \mathrm{~m}$ underground, in the UX15 cavern, and is not accessible during operation due to ionizing radiation. Thus, the control system must allow remote diagnosis. This is ensured by the Detector Control System (DCS) [2], [3], which is responsible for safe and coherent operation of the detector. All ATLAS sub-detectors have their own local DCS, which detailed architecture strongly depends on the structure of the general DCS system of the ATLAS experiment and on electronics architecture and mechanical issues of the sub-detector itself.

\section{BACK END SyStem OF THE TILECAL DCS}

The commercial Supervisory Control And Data Acquisition (SCADA) package ProzessVisualisierungs und Steuerungs System [4] (PVSS II) has been chosen by the Joint COntrols

Manuscript received May 28, 2010. This work was supported in part by FCT, UE, COMPETE and QREN, Portugal, under the contract Project CERN/FP/109323/2009

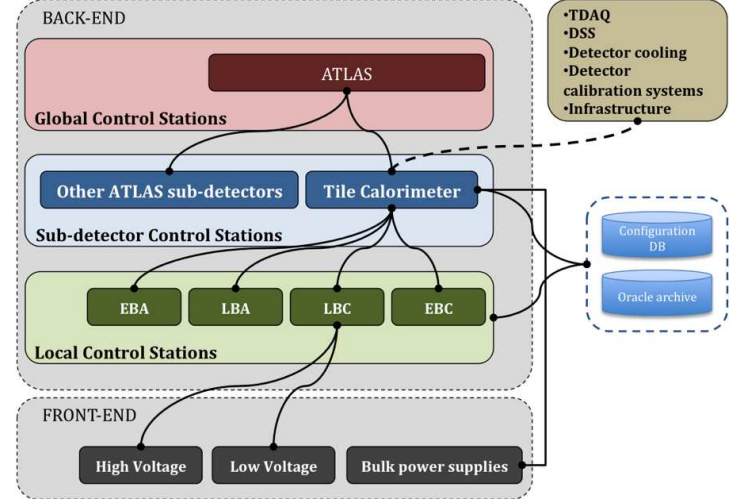

Fig. 1: DCS Hierarchy of the TileCal.

Project (JCOP) [5] at CERN to implement Back End (BE) software for the LHC experiments. The PVSS II is a commercial product, from Austrian company ETM. It is used to connect to hardware devices, acquire data from them, monitor their behavior and to initialize, configure and operate them. PVSS II has a highly distributed and flexible architecture, and it allows connection of several autonomous systems via the network. More details are given in section IV.

The BE system of the ATLAS experiment is organized hierarchically in three layers or levels as shown in figure 1. This hierarchy allows the experiment to be divided in independent partitions, which have the ability to operate in standalone or integrated mode.

At the top layer, there are Global Control Stations (GCS), which are in charge of overall operation of the detector. They provide high level monitoring and control of all sub-detectors, while data processing and command execution are handled at the lower levels. The GCS is available to access all stations in the hierarchy.

The Sub-detector Control Station (SCS) represents the middle level of the hierarchy. The TileCal, as a sub-detector of ATLAS, has its own SCS, which allows the complete operation of the sub-detector, by means of dedicated graphical interfaces. At this level of hierarchy, the connection with the TDAQ system, calibration systems and detector infrastructure takes place in order to ensure that detector operation and physics data taking are synchronized.

The bottom level of the hierarchy is made up of Local Control Stations (LCS), which, in case of the TileCal, handle the low level monitoring and control of LV and HV systems of the sub-detector. The LCS executes the commands received from the layers above and commands issued by itself.

In order to implement the BE system of the TileCal DCS, five rack-mounted computers are used, located in USA15 racks. As it is shown in figure 1 , four of those are used as LCS 
stations (one for each TileCal partition) and one as the SCS station. The operating system of those computers is Windows $\mathrm{XP}$ and they run PVSS II as a system service. The racks are monitored in DCS, as well.

\section{The TIleCal Main DCS Systems}

The TileCal DCS controls and monitors the Low Voltage (LV) power system, the High Voltage (HV) system and monitors the cooling of the electronics. Other control systems do exist for the calibration of the calorimeter: Cs137 source and laser monitoring. These calibration related systems have their own control, but exchange data with the TileCal DCS.

\section{A. The High Voltage Distribution System}

The TileCal High Voltage system is based on HV bulk power supplies located in crates that provide a common high voltage for each set of PMTs. For a common set there is a regulator system, the $\mathrm{HV}$ opto board, that provides fine adjustment of the voltage for each individual PMT over a range of $350 \mathrm{~V}$ below the input voltage. Each set of two HV opto cards is controlled by another board called HV micro card. Each HV micro board controls 68 channels of 5 different types with a reading rate of $0.1 \mathrm{~Hz}$. They are:

- 48 PMTs high voltages;

- 4 input voltages ( $\mathrm{HV}$ in), which corresponds to the quarters of a drawer, supplying 12 PMTs each;

- 7 temperature channels;

- 7 low voltage channels;

- 2 ADC channels.

The communication with the $\mathrm{HV}$ micro card is done via CANbus connected in a daisy-chain. By its turn, the daisychain connects the PC through a Kvaser PCIcan card with 4 ports, which distributes the connection with the modules in 4 branches of 16 drawers each. The communication between the PC and the bulk power supplies is done via RS422 through a TCP/IP gateway. The HV DCS system uses the driver that communicates with the HV micro boards interfaced with a Distributed Information Management, DIM, server [6]. A PVSS DIM client is used to transfer the data and the commands to the SCADA system (see section IV).

The DCS, beyond ensuring the behaviour of the HV system during operation, is also responsible for the monitoring and storing of the applied voltages on the PMTs. The number of monitored parameters is larger than 20000, distributed over 9856 channels. Further, it needs to supply those values to the offline data reconstruction. Photomultipliers gains can change with time and new calibration needs to be applied, so it is necessary to store all the values set during the lifetime of the experiment in a database.

\section{B. The Low Voltage Distribution System}

The low voltage power supplies of the TileCal are located inside the fingers at the extremities of the girder of the modules. They have to provide a total of 8 different voltages to the drawer FE electronics - three for the high voltage system $(\mathrm{HV}-15, \mathrm{HV}+5, \mathrm{HV}+15)$ and five for the motherboard and digitizers (MB-5, MB+5, MB+15, DIG+5, DIG+3.3). The units that produce these individual voltages are called bricks. The voltages and currents are monitored using the electronic modules developed by ATLAS for standard analog and digital I/O - the Embedded Local Monitor Boards, ELMB [7]. Custom made motherboards for the ELMBs were designed for the TileCal LVPS. Auxiliary boards, installed in crates, provide power for the ELMBs' digital and analog circuits and the respective motherboards. It provide also two dedicated current loops in order to switch on/off separately the HV and MB/DIG low voltages. One auxiliary board powers 4 LVPS units.

\section{Cooling system}

The TileCal cooling system operates with water at subatmospheric pressure using a so-called Leakless Cooling System. The system is controlled and monitored locally by a Programmable Logical Controller (PLC). The TileCal cooling plant cools, purifies and provides primary pressure to the water. The cooling plant supplies 24 individual sectors called cooling loops, 6 per TileCal partition. Temperature and pressure of the cooling loops are monitored by the TileCal DCS.

\section{SOFTWARE}

The commercial SCADA package PVSS is the main framework for the ATLAS BE applications [3]. A control station (PC) runs a so-called PVSS "Project" which contains a number of processes called "Managers". Each PVSS Project uses a central database for all current data values, stored in objects called "Datapoints". All Managers have full database access for which PVSS provides transparent synchronization. Data processing is performed in an event-by-event basis using multi-thread callback routines upon value changes. The device-oriented nature of PVSS II permits to model each TileCal module (e.g., a drawer) as a Datapoint (DP), as shown in figure 2. This structure holds a hierarchical and logical representation of the different DCS subsystems of each module. The bottom-most leafs in these structures are the Datapoint Elements (DPE) and represent the actual variables of the subsystems, which can be addressed to the hardware channels.

As an example, figure 3 shows a schematic view of the PVSS interface with the high voltage system.

The Joint COntrols Project (JCOP) Framework [5] is an integrated set of guidelines, components and software tools designed to facilitate the implementation of homogeneous control applications for the LHC experiments using PVSS. The Framework guidelines define a naming convention for PVSS Datapoints, functions, files and standard look of the graphical interfaces of the control system.

\section{DCS DATA ANALYSis}

The data are analysed online by the DCS software, generating warnings and alarms. Some of the alarms can trigger automatic actions for the safety of the detector. The PVSS 


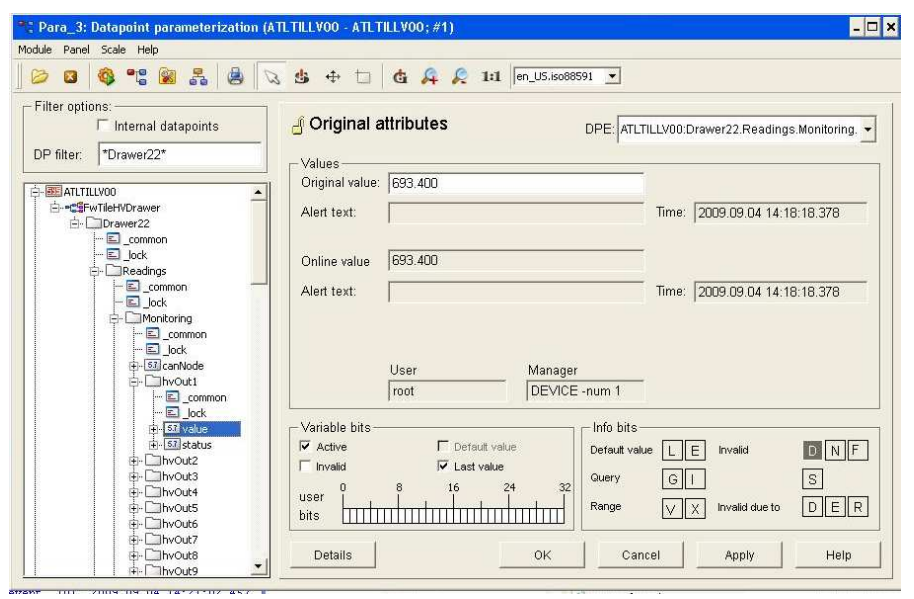

Fig. 2: Example of a drawer Datapoint.

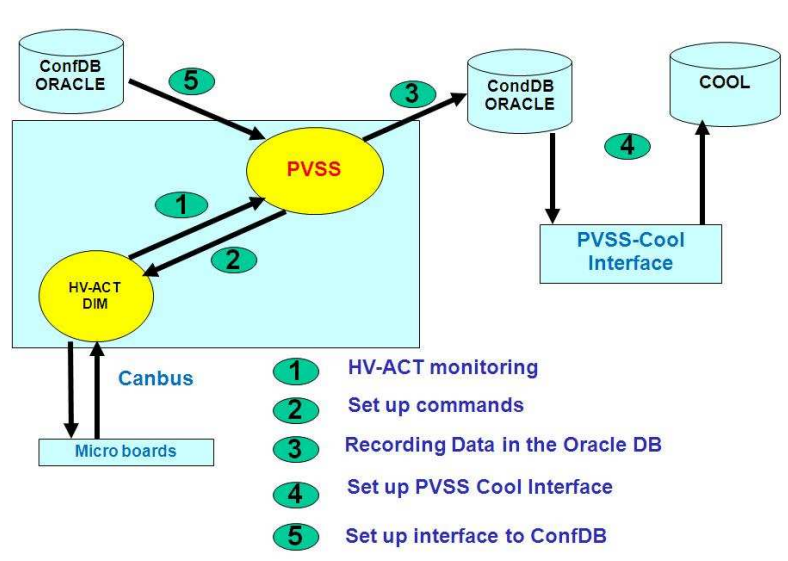

Fig. 3: Schematic view of the interface between the PVSS and the High Voltage system.

trending allows the viewing of historical data from within PVSS applications. Tools are available for the selection of which data should be shown and a trend plot may be displayed. A typical example of a temperature trending plot is shown in figure 4. One should notice that due to data smoothing (see section VIII) the temperature is registered once per hour.

Additionally, offline analyses are done to check and understand detector behaviour and to spot weaknesses of the hardware that may require future intervention. In particular, the analysis performed so far shows that the average stability for the HV of the whole calorimeter is within $0.4 \mathrm{~V}$, which corresponds to $0.4 \%$ reproducibility in the gain of the PMTs (figure 5). Also the high voltage gain dependence on the temperature has been studied, as shown in figure 6. The calorimeter PMTs shows an RMS of $0.2{ }^{\circ} \mathrm{C}$ for a period of 9 months interleaved by a maintenance period.

\section{Finite State Machine}

The Finite State Machine (FSM) toolkit [8], [9] is the top level software for the operation of the detector. It is used to integrate the TileCal in the ATLAS DCS and is used by

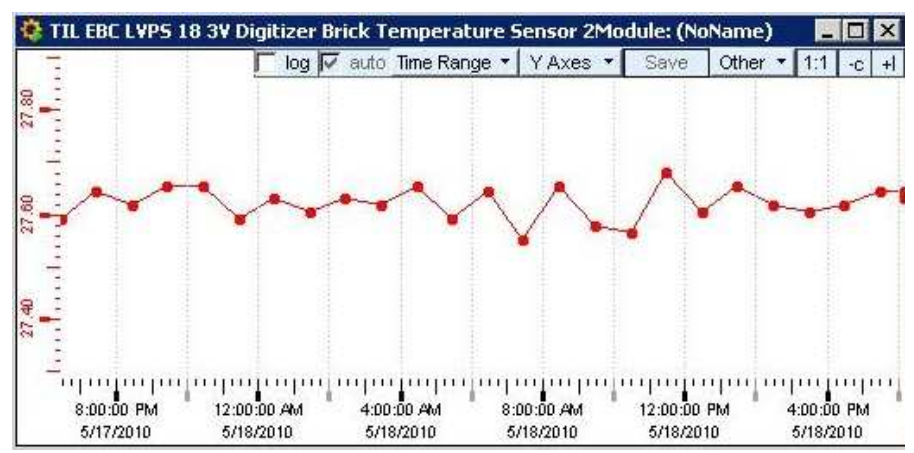

Fig. 4: Display of the temperature trend of a 3.3V digitizer brick during one day. Values are recorded only once per hour or when they change more than a pre-defined threshold.

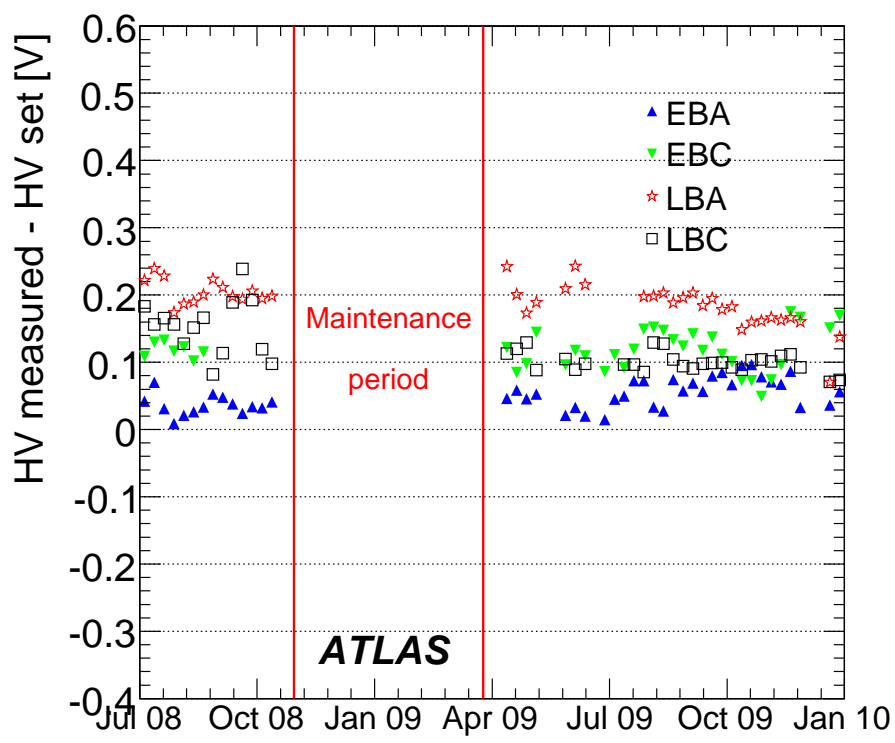

Fig. 5: Stability of the PMT high voltage with respect to its set value, averaging over all PMTs for two periods of 3 and 6 months separated by a maintenance period.

the shifters ${ }^{1}$ for operation. The FSM includes control units, logical units as TileCal modules, and device units. Several operational states (NOT READY, READY, UNKNOWN, etc) are defined and commands implemented on control units allow the transition between them. The Controls hierarchy and the Partitioning rules are implemented based on PVSS and State Management Interface (SMI) [8]. The object model in SMI is described using a dedicated language State Manager Language (SML). PVSS tools are used to configure the system, to log and archive information and to provide User Interfaces. SMI++ tools are used to model Devices and Subsystem behaviour, to automate operations and to recover from error conditions. The basic FSM elements are Device Units (DU) and Control Units

\footnotetext{
${ }^{1}$ Person responsible for the control and monitoring of a sub-detector, or a system, during data taking.
} 


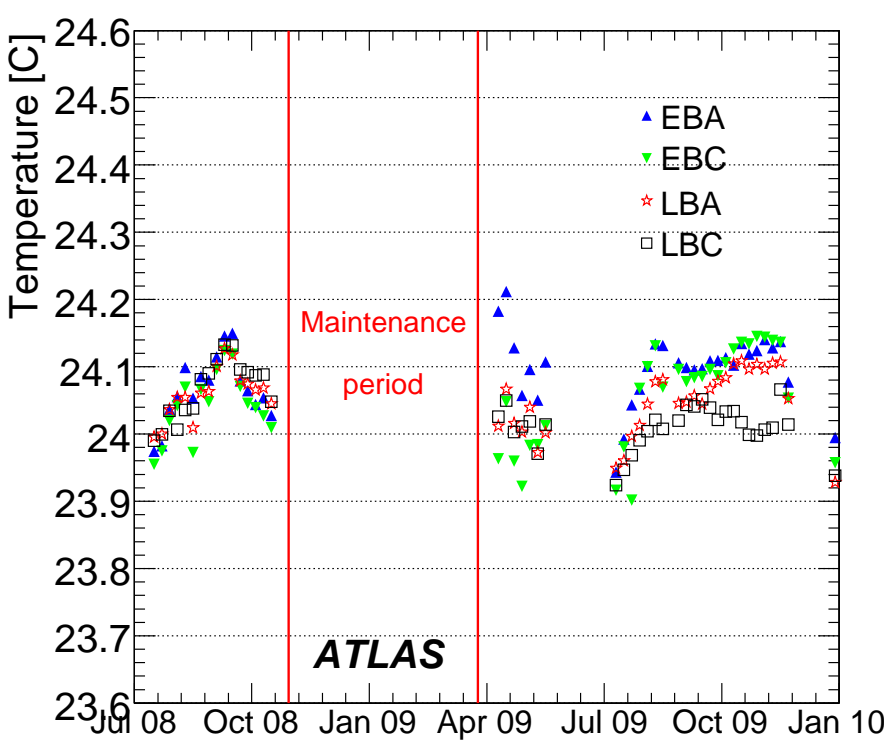

Fig. 6: Temperature stability measured at one PMT per drawer, averaging all values for two periods of 3 and 6 months separated by a maintenance period.

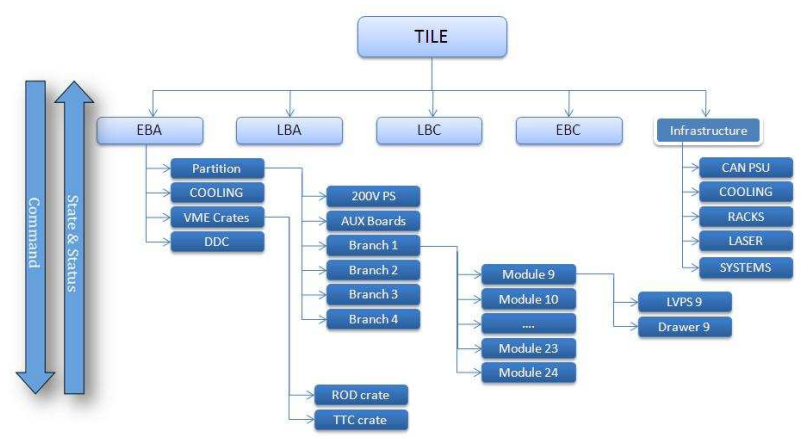

Fig. 7: The TileCal FSM hierarchy.

(CU). The DUs provide the interface with the hardware while the CUs integrate the DUs on the hierarchy. The CUs can be detached from the tree such that they do not propagate their state nor receive commands. The hierarchy of the TileCal FSM tree is shown in figure 7. It does incorporate 21 control units and 600 device units.

\section{Alarm HandLing}

At the ATLAS Global control Station level, there is also available an alert status. The alerts work independently of the FSM, however the status may be closely related. Automatic actions can also be triggered by alarms.

\section{DATA StORING}

The TileCal DCS uses three types of database:

- PVSS ORACLE archive: ORACLE database that stores relevant data for detector behaviour understanding.

- COOL Conditions Database: database which stores relevant data for offline data reconstruction in the ATLAS data analysis software, ATHENA [10].

- Configuration Database: ORACLE database that stores the system structure (lists and hierarchies of devices), device properties, like configuration of archiving and smoothing, and settings, namely output values and alert limits.

The data produced by PVSS are stored in the DCS ORACLE archive, using a Remote Database Manager (RDB). To make this data available in ATHENA, the COOL database is used, which implements an interval of validity database, i.e. objects stored or referenced in COOL have an associated start and end time between which they are valid. COOL data are stored in folders, which are themselves arranged in a hierarchical structure. The PVSS-COOL process takes selected data from the PVSS Oracle Archive and copies it into COOL. During this process the PVSS Datapoints types, that represent devices, are associated with a folder, on which, data from different devices of the same type are allowed.

The original rate of data produced by the TileCal DCS during normal detector operation is too high. To reduce this rate, most of the values are recorded in the database only once per hour or when they change more than a pre-defined threshold (smoothing). The amount of data that is stored is of the order of $300 \mathrm{MB}$ per day.

\section{CONCluSions}

The TileCal DCS is responsible for the monitoring and control of more than 30000 monitored parameters. The system is fully operational and has a major role in the calorimeter performance.

\section{ACKNOWLEDGMENT}

H. Santos thanks to P. Almeida, A. Gomes, S. Nemecek, J. Pina and F. Vinagre for the useful suggestions and fruitful discussions.

\section{REFERENCES}

[1] TileCal Technical Design Report, CERN/LHCC 96-42,(1996); G. Aad et al., The ATLAS Experiment at the CERN Large Hadron Collider, JINST 3 (2008) S08003.

[2] High-Level Triggers, DAQ and DCS Technical Proposal, CERN/LHCC/2000-17 (2000).

[3] A. Barriuso Poy, The detector control system of the ATLAS experiment, 2008 JINST 3 P05006.

[4] ETM professional control GmbH, PVSS II SCADA Product, http://www.pvss.com.

[5] O. Holme, M. Gonzalez Berges, P. Golonka and S. Schmeling, The JCOP framework, proceedings of the 10th International Conference on Accelerator and Large Experimental Physics Control Systems (ICALEPCS 2005), Geneva, Switzerland (2005).

[6] C. Gaspar, M. Dönszelmann and Ph. Charpentier, DIM, a portable, light weight package for information publishing, data transfer and inter-process communication, Comput. Phys. Commun. 140 (2001) 102. 
[7] B. Hallgren, The Embedded Local Monitor Board (ELMB) in the FrontEnd I/O Control System, $7^{\text {th }}$ Workshop for LHC Experiments, Stockholm (2001).

[8] C. Gaspar and B. Franek, Tools for the automation of large distributed control systems, IEEE Trans. Nucl. Sci. 53 (2006) 974.

[9] B. Franek and C. Gaspar, SMI++ - Object Oriented Framework for Designing and Implementing Distributed Control Systems, IEEE Trans. Nucl. Sci. 51 (2004) 513.

[10] ATLAS Collaboration, 'ATHENA The ATLAS Common Framework CERN, 2001. 